\title{
Universality for shape dependence of Casimir effects from Weyl anomaly
}

\author{
Rong-Xin Miao ${ }^{a, 1}$ and Chong-Sun $\mathrm{Chu}^{a, b}$ \\ ${ }^{a}$ Physics Division, National Center for Theoretical Sciences, National Tsing-Hua University, \\ 101 Section 2 Kuang Fu Road, Hsinchu 30013, Taiwan \\ ${ }^{b}$ Department of Physics, National Tsing-Hua University, \\ 101 Section 2 Kuang Fu Road, Hsinchu 30013, Taiwan \\ E-mail: miaorongxin.physics@gmail.com, cschu@phys.nthu.edu.tw
}

Abstract: We reveal elegant relations between the shape dependence of the Casimir effects and Weyl anomaly in boundary conformal field theories (BCFT). We show that for any BCFT which has a description in terms of an effective action, the near boundary divergent behavior of the renormalized stress tensor is completely determined by the central charges of the theory. These relations are verified by free BCFTs. We also test them with holographic models of BCFT and find exact agreement. We propose that these relations between Casimir coefficients and central charges hold for any BCFT. With the holographic models, we reproduce not only the precise form of the near boundary divergent behavior of the stress tensor, but also the surface counter term that is needed to make the total energy finite. As they are proportional to the central charges, the near boundary divergence of the stress tensor must be physical and cannot be dropped by further artificial renormalization. Our results thus provide affirmative support on the physical nature of the divergent energy density near the boundary, whose reality has been a long-standing controversy in the literature.

Keywords: AdS-CFT Correspondence, Classical Theories of Gravity, Conformal Field Theory

ARXIV EPRINT: 1706.09652

\footnotetext{
${ }^{1}$ Corresponding author.
} 


\section{Contents}

1 Introduction 1

2 Shape dependence of Casimir effects from Weyl anomaly 2

3 Free and holographic BCFT 4

4 Conclusions and discussions $\quad 8$

$\begin{array}{ll}\text { A Solutions to holographic BCFT } & 9\end{array}$

$\begin{array}{lll}\text { A.1 3d BCFT } & 9\end{array}$

$\begin{array}{lll}\text { A.2 } & 4 \mathrm{~d} \text { BCFT } & 11\end{array}$

\section{Introduction}

The Casimir effect [1] originates from the effect of boundary on the zero point energymomentum of quantized fields in a system. As a fundamental property of the quantum vacuum, it has important consequences on the system of concern and has been applied to a wide range of physical problems, such as classic applications in the study of the Casimir force between conducting plates (and nano devices) [2, 3], dynamical compactification of extra dimensions in string theory $[4,5]$, candidate of cosmological constant and dark energy, ${ }^{1}$ as well as dynamical Casimir effect and its applications. ${ }^{2}$

The near boundary behavior of the stress tensor of a system is crucial to the understanding of the Casimir effect. For a Quantum Field Theory (QFT) on a manifold $M$ of integer dimension $d$ and boundary $P$, the renormalized stress tensor is divergent near the boundary [8]:

$$
\left\langle T_{i j}\right\rangle=x^{-d} T_{i j}^{(d)} \ldots+x^{-1} T_{i j}^{(1)}, \quad x \sim 0,
$$

where $x$ is the proper distance from the boundary and $T_{i j}^{(n)}$ with $n \geq 1$ depend only on the shape of the boundary and the kind of QFT under consideration. For CFT with conformal invariant boundary condition (BCFT), one further require that divergent parts of renormalized stress tensor are traceless in order to get a well-defined finite Weyl anomaly without divergence. It is also natural to impose the conservation condition of energy:

$$
\lim _{x \rightarrow 0}\left\langle T_{i}^{i}\right\rangle=O(1), \quad \nabla_{i}\left\langle T_{j}^{i}\right\rangle=0
$$

\footnotetext{
${ }^{1}$ See for example ref. [6].

${ }^{2}$ See for example ref. [7].
} 
Substituting (1.1) into the above equations, [8] obtains

$$
\begin{gathered}
T_{i j}^{(d)}=0, \quad T_{i j}^{(d-1)}=2 \alpha_{1} \bar{k}_{i j}, \\
T_{i j}^{(d-2)}=\frac{-4 \alpha_{1}}{d-1} n_{(i} h_{j)}^{l} \nabla_{l} k-\frac{4 \alpha_{1}}{d-2} n_{(i} h_{j)}^{l} n^{p} R_{l p} \\
\quad+\frac{2 \alpha_{1}}{d-2}\left(n_{i} n_{j}-\frac{h_{i j}}{d-1}\right) \operatorname{Tr} \bar{k}^{2}+t_{i j}, \\
t_{i j}:=\left\lceil\beta_{1} C_{i k j l} n^{k} n^{l}+\beta_{2} \mathcal{R}_{i j}+\beta_{3} k k_{i j}+\beta_{4} k_{i}^{l} k_{l j}\right\rceil,
\end{gathered}
$$

where $n_{i}, h_{i j}$ and $\bar{k}_{i j}$ are respectively the normal vector, induced metric and the traceless part of extrinsic curvature of the boundary $P$. The tensor $t_{i j}$ is tangential: $n^{i} t_{i j}=0,\lceil\rceil$ denotes the traceless part, $C_{i j k l}$ is Weyl tensor of $M$ and $\mathcal{R}_{i j}$ is the intrinsic Ricci tensor of $P$. The coefficients $\left(\alpha, \beta_{i}\right)$ fixes the shape dependence of the leading and subleading Casimir effects of BCFT. The main goal of this letter is to show that one can fix completely these Casimir coefficients in terms of the bulk and boundary central charges.

\section{Shape dependence of Casimir effects from Weyl anomaly}

Consider a BCFT with a well defined effective action. The Weyl anomaly $\mathcal{A}$, defined as the trace of renormalized stress tensor, can be obtained as the logarithmic UV divergent term of the effective action,

$$
I=\cdots+\mathcal{A} \log \left(\frac{1}{\epsilon}\right)+I_{\text {finite }}
$$

where $\cdots$ denotes terms which are UV divergent in powers of the UV cutoff $1 / \epsilon$, and $I_{\text {finite }}$ is the renormalized, UV finite part of the effective action. This part is dependent on the subtraction scheme. But the dependence is irrelevant for the discussion below and our results hold for any renormalization scheme.

Inspired by [9, 10], let us regulate the effective action by excluding from its volume integration a small strip of geodesic distance $\epsilon$ from the boundary. Then there is no explicit boundary divergences in this form of the effective action, however there are boundary divergences implicit in the bulk effective action which is integrated up to distance $\epsilon$. The variation of effective action is given by

$$
\delta I=\frac{1}{2} \int_{x \geq \epsilon} \sqrt{g} \hat{T}^{i j} \delta g_{i j}
$$

where $\hat{T}^{i j}=\frac{2 \delta I}{\sqrt{g} \delta g_{i j}}$ is the non-renormalized bulk stress tensor. The renormalized bulk stress tensor is defined by the difference of the non-renormalized bulk stress tensor against a reference one [8]:

$$
T^{i j}=\hat{T}^{i j}-\hat{T}_{0}^{i j},
$$

where $\hat{T}_{0}^{i j}$ is the non-renormalized stress tensor defined for the same CFT without boundary. It is

$$
\delta I_{0}=\frac{1}{2} \int_{x \geq \epsilon} \sqrt{g} \hat{T}_{0}^{i j} \delta g_{i j}
$$


where $I_{0}$ is the effective action of the CFT with the boundary removed, hence the integration over the region $x \geq \epsilon$. Subtract (2.4) from (2.2) and focus on only the logarithmically divergent terms, we obtain our key formula

$$
(\delta \mathcal{A})_{\partial M}=\left(\frac{1}{2} \int_{x \geq \epsilon} \sqrt{g} T^{i j} \delta g_{i j}\right)_{\log (1 / \epsilon)},
$$

where $(\delta \mathcal{A})_{\partial M}$ is the boundary terms in the variations of Weyl anomaly and $T^{i j}$ is the renormalized bulk stress tensor. In the above derivations, we have used the fact that $I$ and $I_{0}$ have the same bulk Weyl anomaly so that

$$
(\delta \mathcal{A})_{\partial M}=\left(\delta I-\delta I_{0}\right)_{\log (1 / \epsilon)}
$$

We observe that as the right hand side of (2.5) must give an exact variation, this imposes strong constraints on the possible form of the stress tensor near the boundary since this is where one would pick up logarithmic divergent contribution on integration near the boundary. It is this integrability of the variations which helps us to fix the Casimir effects in terms of the Weyl anomaly. To proceed, let us start with the metric written in the Gauss normal coordinates

$$
d s^{2}=d x^{2}+\left(h_{a b}-2 x k_{a b}+x^{2} q_{a b}+\cdots\right) d y^{a} d y^{b},
$$

where $x \in[0,+\infty)$. The coefficients $k_{a b}, q_{a b}, \cdots$ parametrize the derivative expansion (with respect to both $x$ and $y^{a}$ ) of the metric. Consider variation of the metric with $\delta g_{x i}=0$ and $\delta g_{a b}=\delta h_{a b}-2 x \delta k_{a b}+\cdots$. Take first the $3 \mathrm{~d}$ BCFT as an example. The Weyl anomaly of $3 \mathrm{~d}$ BCFT is given by [11]

$$
\mathcal{A}=\int_{P} \sqrt{h}\left(b_{1} \mathcal{R}+b_{2} \operatorname{Tr} \bar{k}^{2}\right),
$$

where $b_{1}, b_{2}$ are boundary central charges which depends on the boundary conditions. Taking the variation of (2.8), we have

$$
b_{2} \int_{P} \sqrt{h}\left[\left(\frac{\operatorname{Tr} \bar{k}^{2}}{2} h^{a b}-2 \bar{k}_{c}^{a} k^{c b}\right) \delta h_{a b}+2 \bar{k}^{a b} \delta k_{a b}\right] .
$$

Now we turn to calculate the variation of Weyl anomaly from the last term of (2.5). Note that $C_{i j k l}=\left\lceil\mathcal{R}_{i j}\right\rceil=0$ for $d=3$. Note also that $\bar{k}_{i j}(x)=g_{i}^{i^{\prime}} g_{j}^{j^{\prime}} \bar{k}_{i^{\prime} j^{\prime}}(0)=\bar{k}_{i j}(0)-2 x k_{(i}^{l} \bar{k}_{j) l}+$ $O\left(x^{2}\right)$, where $g_{i}^{i^{\prime}}$ is the bivector of parallel transport between $x$ and $x=0$ [8]. Taking these facts into account and substitute (1.1) and (1.3) into the last term of (2.5), integrate over $x$ and select the logarithmic divergent term, we obtain

$$
\begin{aligned}
-\alpha_{1} & \int_{P} \sqrt{h}\left[\left(\frac{\operatorname{Tr} \bar{k}^{2}}{2} h^{a b}-2 \bar{k}_{c}^{a} k^{c b}\right) \delta h_{a b}+2 \bar{k}^{a b} \delta k_{a b}\right] \\
& +\int_{P} \sqrt{h}\left[\left(\frac{\beta_{3}}{2}-\alpha_{1}\right) k \bar{k}^{a b} \delta h_{a b}+\frac{\beta_{4}}{2}\left\lceil k_{c}^{a} k^{c b}\right\rceil \delta h_{a b}\right] .
\end{aligned}
$$

Note that (2.10) is made up of a structure of curvature components different from those appearing in (2.9). Integrability of (2.10) gives $\beta_{3}=2 \alpha_{1}$ and $\beta_{4}=0$. Comparing (2.9) 
with (2.10) gives $\alpha_{1}=-b_{2}$. All together, we obtain the relations between the Casimir coefficients of the stress tensor and the boundary central charges:

$$
\alpha_{1}=-b_{2}, \quad \beta_{3}=-2 b_{2}, \quad \beta_{4}=0 .
$$

Similarly for 4d BCFT, we can obtain the shape dependence of Casimir effects from the Weyl anomaly $[12,13]$

$$
\begin{aligned}
\mathcal{A}= & \int_{M} \sqrt{g}\left(\frac{c}{16 \pi^{2}} C^{i j k l} C_{i j k l}-\frac{a}{16 \pi^{2}} E_{4}\right) \\
& +\int_{P} \sqrt{h}\left(b_{3} \operatorname{Tr} \bar{k}^{3}+b_{4} C^{a c}{ }_{b c} \bar{k}_{a}^{b}\right),
\end{aligned}
$$

where $a, c$ are bulk central charges and $b_{3}, b_{4}$ are boundary central charges. $E_{4}$ is the Euler density including the boundary term. To derive $t_{i j}$, we set $\delta h_{i j}=0$ for simplicity, since it only affects the third order derivative terms in the stress tensor. Taking variation of (2.12) and comparing the boundary term with the last term of (2.5), we obtain

$$
\begin{aligned}
& \alpha_{1}=\frac{b_{4}}{2}, \quad \beta_{1}=\frac{c}{2 \pi^{2}}+b_{4}, \quad \beta_{2}=0, \\
& \beta_{3}=2 b_{3}+\frac{13}{6} b_{4}, \quad \beta_{4}=-3 b_{3}-2 b_{4} .
\end{aligned}
$$

It is remarkable that the boundary behavior of the stress tensor is completely determined by the boundary and bulk central charges However, it is independent of the central charge related to Euler density due to the fact that topological invariants do not change under local variations. We propose that the relations (2.11) and (2.13) between Casimir coefficients and central charges hold for general BCFT.

\section{Free and holographic BCFT}

Let us verify our general statements with free BCFT. The renormalized stress tensor of $4 \mathrm{~d}$ free BCFT has been calculated in $[8,14,15]$. The bulk and boundary central charges for $4 \mathrm{~d}$ free BCFTs were obtained in [12]. We summary these results in table 1 and table 2. Note that the results for Maxwell field apply to both absolute and relative B.C. We find these data obey exactly the relations (2.13). $\beta_{1}$ for Maxwell field is absence in the literature. Here from (2.13), we predict that $\beta_{1}=0$ for all $4 \mathrm{~d}$ free BCFT due to the fact that $c=-2 \pi^{2} b_{4}$ for $4 \mathrm{~d}$ free BCFT. As we will show below, this relation is violated by strongly-coupled CFT dual to gravity. As a result, $\beta_{1}$ is non-zero in general. Comparing with [15], we note that there is a minus sign typo of $\beta_{4}$ for Maxwell field in [8].

Now let us investigate the shape dependence of Casimir effects in holographic models of BCFT. Consider a BCFT defined on a manifold $M$ with a boundary $P$. Takayanagi [16] proposed to extend the $d$ dimensional manifold $M$ to a $d+1$ dimensional asymptotically AdS space $N$ so that $\partial N=M \cup Q$, where $Q$ is a $d$ dimensional manifold which satisfies $\partial Q=\partial M=P$. The gravitational action for holographic BCFT is [16] $\left(16 \pi G_{N}=1\right)$

$$
I=\int_{N} \sqrt{G}(R-2 \Lambda)+2 \int_{Q} \sqrt{\gamma}(K-T)
$$




\begin{tabular}{|c|c|c|c|c|c|}
\hline & $\alpha_{1}$ & $\beta_{1}$ & $\beta_{2}$ & $\beta_{3}$ & $\beta_{4}$ \\
\hline Scalar, Dirichlet B.C & $-\frac{1}{480 \pi^{2}}$ & 0 & 0 & $-\frac{19}{10080 \pi^{2}}$ & $-\frac{1}{420 \pi^{2}}$ \\
\hline Scalar, Robin B.C & $-\frac{1}{480 \pi^{2}}$ & 0 & 0 & $-\frac{1}{288 \pi^{2}}$ & 0 \\
\hline Maxwell field & $-\frac{1}{40 \pi^{2}}$ & $(0)$ & 0 & $-\frac{43}{840 \pi^{2}}$ & $\frac{1}{70 \pi^{2}}$ \\
\hline
\end{tabular}

Table 1. Casimir coefficients for $4 \mathrm{~d}$ free BCFT.

\begin{tabular}{|c|c|c|c|c|}
\hline & $a$ & $c$ & $b_{3}$ & $b_{4}$ \\
\hline Scalar, Dirichlet B.C & $\frac{1}{360}$ & $\frac{1}{120}$ & $\frac{1}{280 \pi^{2}}$ & $-\frac{1}{240 \pi^{2}}$ \\
\hline Scalar, Robin B.C & $\frac{1}{360}$ & $\frac{1}{120}$ & $\frac{1}{360 \pi^{2}}$ & $-\frac{1}{240 \pi^{2}}$ \\
\hline Maxwell field & $\frac{31}{180}$ & $\frac{1}{10}$ & $\frac{1}{35 \pi^{2}}$ & $-\frac{1}{20 \pi^{2}}$ \\
\hline
\end{tabular}

Table 2. Central charges for $4 \mathrm{~d}$ free BCFT.

plus terms on $M$ and $P$. Here $T$ is a constant which can be regarded as the holographic dual of boundary conditions of BCFT $[17,18]$. A central issue in the construction of the AdS/BCFT is the determination of the location of $Q$ in the bulk. [16] propose to use the Neumann boundary condition

$$
K_{\alpha \beta}-(K-T) \gamma_{\alpha \beta}=0
$$

to fix the position of $Q$. In $[17,18]$ we found there is generally no solution to (3.2) for bulk metric that arose from the FG expansion of a general non-symmetric boundary. The reason is because $Q$ is of co-dimension one and we only need one condition to determine it's position, while there are too many extra conditions in (3.2). To resolve this, we suggested in $[17,18]$ to use the trace of $(3.2),(1-d) K+d T=0$, to determine the position of $Q$. Nonetheless, it is also possible that one may need to relax the assumption that the bulk metric admits a valid FG expansion, as has been attempted in [20] for some nonsymmetric boundary in $\mathrm{BCFT}_{3}$. In contrast to a FG-expanded metric whose form near the boundary $M$ is completely fixed, a non-FG expanded metric has more degree of freedom. It was suggested in [20] that the embedding equation (3.2) may admit a solution if the bulk metric is also allowed to adjust itself. However in general this is a highly non-trivial problem and there is no systematic method available to construct gravity solutions for BCFT in general dimensions $d$ and with an arbitrary non-symmetric boundary $\left(\bar{k}_{a b} \neq 0\right)$ that is not FG expanded. Remarkably this problem can solved and we will now present the solution.

To make progress in this front, we find that one can instead consider an expansion in powers of small derivatives of the metric and keep both the $z$ and $x$ dependence as exact to construct a perturbative solution to the Einstein equation. For simplicity, we consider the case of $h_{a b}=\delta_{a b}$ here. The more general case of a nontrivial boundary metric can be analysed. We comment on this in the supplementary information. We find useful to consider the following metric ansatz

$$
d s^{2}=\frac{d z^{2}+d x^{2}+\left(\delta_{a b}-2 x \bar{k}_{a b} f\right) d y^{a} d y^{b}}{z^{2}}+\cdots
$$




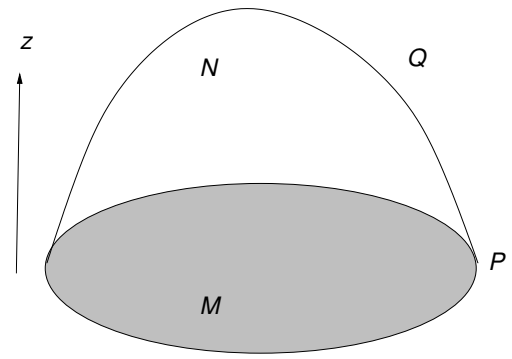

Figure 1. BCFT on $M$ and its dual $N$.

with $f=f(x, z)$ a function such that $f(x, 0)=1$. To find solution, let us first consider the region $x \geq 0$ and consider the ansatz $f=f(z / x)$. This ansatz plays an important role to solve (3.2) for non-symmetric boundary with $\bar{k}_{a b} \neq 0$. For simplicity we consider a traceless $k_{a b}=\bar{k}_{a b}$ extrinsic curvature here. The solution for the general case is given in the supplementary information. Substituting (3.3) into Einstein equation and writing $s:=z / x>0$, we obtain at the order $O(k)$ a single equation

$$
s\left(s^{2}+1\right) f^{\prime \prime}(s)-(d-1) f^{\prime}(s)=0 .
$$

It has the solution

$$
f(s)=1-\alpha_{1} \frac{s^{d}{ }_{2} F_{1}\left(\frac{d-1}{2}, \frac{d}{2} ; \frac{d+2}{2} ;-s^{2}\right)}{d} .
$$

To obtain a solution of the Einstein equation for $x<0$, one may analytic continuate (3.5) to the region $s<0$. However this solution while continuous at $s=0$, is discontinuous at $x=0$ as the region near $x=0$ is mapped to widely separated regions $s= \pm \infty$. Another possibility is to first rewrite the expression (3.5) in terms of $x$ and $z$, and then analytic continuate the resulting function $f(x, z)$ to the region $x<0$. In this way, we obtain a solution of the Einstein equation that is continuous at $x=0$. For example, for $d=3$, we have

$$
\begin{aligned}
& f(x, z)=1-\alpha_{1}\left(\frac{z}{x}-g(x, z)\right) \\
& g(x, z)=\frac{\pi}{2}-2 \tan ^{-1}\left(x /\left(z+\sqrt{z^{2}+x^{2}}\right)\right) .
\end{aligned}
$$

Let us make some comments. 1 . For general $d$, the perturbation $2 x \bar{k}_{a b} f(x, z)$ is finite which shows that (3.4) is a well-defined metric. 2. Note that formally one can expand $f$ as a power series of $z$ and interpret that as a FG expansion of the metric (3.3). However the series does not converge whenever $x<z$. Therefore for the boundary $(x \rightarrow 0)$ physics we are interested in, it is necessary to use the exact solution without performing the FG expansion. 3. The perturbative background (3.3), (3.5) to the Einstein equation is an interesting result which may be useful for other studies as well.

So far the coefficient $\alpha_{1}$ is arbitrary. If we now consider (3.2) in this background, we find that one can solve the embedding function of $Q$ as $x=-\sinh (\rho) z+O\left(k^{2}\right)$ provided that $\alpha_{1}$ is fixed at the same time. Please see the supplementary information for more details. See table 3 for values of $\alpha_{1}$ obtained from holography, where we have re-parametrized 


\begin{tabular}{|c|c|c|c|c|c|}
\hline & $\alpha_{1}$ & $\beta_{1}$ & $\beta_{2}$ & $\beta_{3}$ & $\beta_{4}$ \\
\hline $3 \mathrm{~d}$ & $\frac{-1}{\theta}$ & 0 & 0 & $\frac{-2}{\theta}$ & 0 \\
\hline $4 \mathrm{~d}$ & $\frac{-1}{2(1+\tanh \rho)}$ & $\frac{\tanh \rho}{\tanh \rho+1}$ & 0 & $\frac{5+4 \tanh \rho}{-6(1+\tanh \rho)}$ & $\frac{\tanh \rho}{\tanh \rho+1}$ \\
\hline
\end{tabular}

Table 3. Casimir coefficients for holographic stress tensor.

$T=(d-1) \tanh \rho$ and $\theta=\frac{\pi}{2}+2 \tan ^{-1}\left(\tanh \left(\frac{\rho}{2}\right)\right)$ is the angle between $M$ and the bulk boundary $Q$. Using (3.3), (3.5), we can derive the holographic stress tensor [21]

$$
T_{i j}=\lim _{z \rightarrow 0} d \frac{\delta g_{i j}}{z^{d}}=2 \alpha_{1} \frac{\bar{k}_{i j}}{x^{d-1}}+O\left(k^{2}\right),
$$

which takes the expected form (1.3a). According to [21], $T_{i j}$ (3.7) automatically satisfy the traceless and divergenceless conditions (1.2). Note that in general the stress tensor (3.7) also contains contributions from $\left.g_{i j}\right|_{z=0}$ in even dimensions [21]. However, these contributions are finite, so we can ignore them without loss of generality since we focus on only the divergent parts in this letter.

Similarly, we can work out the next order solutions to both the Einstein equation and (3.2), and then derive the stress tensor up to the order $O\left(k^{2}\right)$ by applying the formula (3.7). See the appendix for details. It turns out that the holographic stress tensor takes exactly the expected expression (1.3) with the coefficients listed in table 3. These coefficients indeed satisfy the relations $(2.11),(2.13)$ provided the boundary central charges are given $b y^{3}$

$$
\begin{aligned}
& b_{2}=\frac{1}{\theta}, \\
& b_{3}=\frac{1}{1+\tanh \rho}-\frac{1}{3}, \quad b_{4}=\frac{-1}{1+\tanh \rho},
\end{aligned}
$$

for $3 \mathrm{~d}$ and $4 \mathrm{~d}$ respectively. Since we have many more relations (8) than unknown variables (3), this is a non-trivial check of the universal relations (2.11), (2.13) as well as for the holographic proposal (3.2). In fact, the central charges (3.8a), (3.8b) can be independently derived from the logarithmic divergent term of action by using the perturbation solution of order $O\left(k^{d-1}\right)$. One can consider general boundary conditions by adding intrinsic curvatures on $Q$ [18]. In this case the boundary central charges change but the relations (2.11), (2.13) remain the same. We can also reproduce these relations in the holographic model $[17,18]$. These are all strong supports for the universal relations (2.11), (2.13). The fact that the both the holographic models of $[16]$ and ours $[17,18]$ verify the universal relations (2.11), (2.13) suggests that both proposals are consistent holographic models of BCFT. We remark that in general there could be more than one self-consistent boundary conditions for a theory [19] and so there is no contradiction between [16] and $[17,18]$. This is supported by the fact that the two holographic models gives different boundary central charges despite the same universal relations are satisfied.

\footnotetext{
${ }^{3}$ Note that in [18] we find $b_{2}$ and $b_{4}$ of [16] vanish for bulk solution that can be FG expanded. Here we clarify that $b_{2}$ and $b_{4}$ of [16] can be non-zero in general if one employ the new background (3.3) in the non-FG expanded regime.
} 
From holographic BCFT [16-18], we can also gain some insight into the total energy. Applying the holographic renormalization of BCFT $[17,18]$, we obtain the total stress tensor:

$$
T_{i j}=2 \alpha_{1} \frac{\bar{k}_{i j}}{x^{d-1}}-\delta(x ; P) \frac{2 \alpha_{1}}{d-2} \frac{\bar{k}_{i j}}{\epsilon^{d-2}}+O\left(k^{2}\right), \quad x \sim \epsilon .
$$

Note that the first term, a local energy density, give rises to a divergence in the total energy that cannot be canceled with any local counterterm in the BCFT, but only with the inclusion of the second term, a surface counterterm as first constructed in [14]. The surface counterterm is localized at the boundary surface $P$, which has been shifted from $x=0$ to a position $x=\epsilon$. The requirement of finite energy fixes [14] the relative coefficients of the two terms in (3.9). Remarkably the holographic constructions [16-18] reproduce precisely also the surface counter term with the needed coefficient to make the total energy finite : $\int_{\epsilon}^{\infty} d x T_{i j}=O\left(k^{2}\right)<\infty$, which agrees with the results of $[14,22]$.

\section{Conclusions and discussions}

In this letter, we have shown that with the help of an effective action description, the divergent parts of the stress tensor of a BCFT is completely determined by the central charges of the theory. The found relations between the Casimir coefficients and the central charges are verified by free BCFT as well as holographic models of BCFT. We propose that these relations hold universally for any BCFT. Using the holographic models, we also reproduce remarkably the precise surface counterterm that is needed to render the total energy of the BCFT finite.

Our results are useful for the study of shape dependence of Casimir effects [23-25] and the theory of BCFT $[26,27]$. For Casimir effects where there are spacetime on both sides of the boundary, it has been argued that the divergent stress tensor originates from the unphysical nature of classical "perfect conductor" boundary conditions [8]. In reality there would be an effective cut off $\epsilon$ below which the short wavelength vibrational modes do not "see the boundary". However for BCFT where there is no spacetime outside the boundary, the divergent one point function of stress tensor is expected and physical. According to $[28],{ }^{4}$ one can derive the one point function of an operator in BCFT from the two point functions of operators in CFT by using the mirror method. Since two point functions are divergent when two points are approaching, it is not surprising that the one point function of BCFT diverge near the boundary. This is due to the interaction with the boundary, or equivalently, the mirror image. Note that although the stress tensor diverges, the total energy is finite. Thus BCFT is self-consistent.

Our discussions can be generalized to higher dimensions naturally. Furthermore, our discussions also apply to defect conformal field theory (DCFT) [29] with general codimensions, which is a problem of great interest. For example, the case of codimension 2 DCFT is related to the shape dependence of Rényi entropy [9, 10, 30-33]. It is interesting to see whether the spirit of this letter can apply to general QFT. It is also very interesting to

\footnotetext{
${ }^{4}$ We thank John Cardy for [28].
} 
generalize and apply the techniques of the holographic models to study the expectation value of current in boundary systems, e.g. edge current of topological materials.

\section{Acknowledgments}

We thank John Cardy, WuZhong Guo, Hugh Osborn and Douglas Smith for useful discussions and comments. This work is supported in part by NCTS and the grant MOST 105-2811-M-007-021 of the Ministry of Science and Technology of Taiwan.

\section{A Solutions to holographic BCFT}

Here we give details about solutions to the Einstein equations and the boundary conditions (3.2) to the next order in derivative expansion of the boundary metric (i.e. $O\left(k^{2}\right)$ in the case of a flat boundary metric $h_{a b}=\delta_{a b}$ ). Consider the following ansatz for $x>0$,

$$
\begin{aligned}
d s^{2}= & \frac{1}{z^{2}}\left[d z^{2}+\left(1+x^{2} X\left(\frac{z}{x}\right)\right) d x^{2}\right. \\
& \left.\quad+\left(\delta_{a b}-2 x \bar{k}_{a b} f\left(\frac{z}{x}\right)-2 x \frac{k}{d-1} \delta_{a b}+x^{2} Q_{a b}\left(\frac{z}{x}\right)\right) d y^{a} d y^{b}\right] \\
& +O\left(k^{3}\right),
\end{aligned}
$$

where the functions $X\left(\frac{z}{x}\right)$ and $Q_{a b}\left(\frac{z}{x}\right)$ are of order $O\left(k^{2}\right)$. We require that

$$
f(0)=1, \quad X(0)=0, \quad Q_{a b}(0)=q_{a b}
$$

so that the metric of BCFT takes the form (2.7) in Gauss normal coordinates.

\section{A.1 3d BCFT}

Let us first study the case $d=3$. The generalization to higher dimensions is straightforward. For simplicity, we further set $k_{a b}=\operatorname{diag}\left(k_{1}, k_{2}\right), q_{a b}=\operatorname{diag}\left(q_{1}, q_{2}\right)$, where $k_{a}, q_{a}$ are constants. Substituting (A.1) into the Einstein equations, and using (A.2) to fix the integral constants, we obtain (3.5) and

$$
\begin{aligned}
& f(s)=1-\alpha_{1}(s-g(s)) \\
& \begin{aligned}
Q_{11}(s)=\frac{1}{8}[ & 4 q_{1}\left(s^{2}+2\right)-\alpha_{1}^{2}\left(k_{1}-k_{2}\right)^{2}\left(s^{2}-3\right) g(s)^{2} \\
& -2 \alpha_{1}^{2}\left(k_{1}-k_{2}\right)^{2} \log \left(s^{2}+1\right)+s\left(5 \alpha_{1}^{2}\left(k_{1}-k_{2}\right)^{2} s+4 \alpha_{2}\right) \\
& +s\left(2 \alpha_{1}\left(-5 k_{1}^{2}+8 k_{2} k_{1}+k_{2}^{2}\right)-4 s\left(k_{1}^{2}-k_{2} k_{1}-k_{2}^{2}+q_{2}\right)\right) \\
& -2 g(s)\left(\alpha_{1} k_{1}^{2}\left(3 \alpha_{1} s+s^{2}-5\right)+2 \alpha_{2}\left(s^{2}+1\right)\right) \\
& \left.\quad-2 \alpha_{1} g(s)\left(k_{2}^{2}\left(3 s\left(\alpha_{1}+s\right)+1\right)+2 k_{1} k_{2}\left(4-3 \alpha_{1} s\right)\right)\right] \\
Q_{22}(s)=\frac{1}{8}[ & 4 q_{2}\left(s^{2}+2\right)-\alpha_{1}^{2}\left(k_{1}-k_{2}\right)^{2}\left(s^{2}-3\right) g(s)^{2} \\
& +s\left(5 \alpha_{1}^{2}\left(k_{1}-k_{2}\right)^{2} s-4 \alpha_{2}\right)-2 \alpha_{1}^{2}\left(k_{1}-k_{2}\right)^{2} \log \left(s^{2}+1\right) \\
& +s\left(4 s\left(k_{1}^{2}+k_{2} k_{1}-k_{2}^{2}-q_{1}\right)-2 \alpha_{1}\left(k_{1}^{2}-4 k_{2} k_{1}+7 k_{2}^{2}\right)\right)
\end{aligned}
\end{aligned}
$$




$$
\begin{aligned}
& +2 g(s)\left(2 \alpha_{2}\left(s^{2}+1\right)-\alpha_{1} k_{1}^{2}\left(3 \alpha_{1} s+s^{2}-1\right)\right) \\
& \left.+2 \alpha_{1} g(s)\left(k_{2}^{2}\left(-3 \alpha_{1} s+s^{2}+7\right)+2 k_{1} k_{2}\left(3 \alpha_{1} s+2 s^{2}-2\right)\right)\right], \\
X(s)=\frac{1}{4}[ & -\alpha_{1}^{2}\left(k_{1}-k_{2}\right)^{2} s^{2} \log \left(s^{2}+1\right)-2 \alpha_{1}\left(k_{1}-k_{2}\right)^{2} s \\
& +\alpha_{1}\left(k_{1}-k_{2}\right)^{2} g(s)\left(\alpha_{1}\left(s^{2}+1\right) g(s)+2 s\left(s-\alpha_{1}\right)+2\right) \\
& \left.+s\left(\alpha_{1}^{2}\left(k_{1}-k_{2}\right)^{2} s-2 s\left(k_{1}^{2}+k_{2} k_{1}+k_{2}^{2}-q_{1}-q_{2}\right)\right)\right],
\end{aligned}
$$

where $s=z / x$ and $g(s)=\frac{\pi}{2}-2 \tan ^{-1}\left(1 /\left(s+\sqrt{s^{2}+1}\right)\right)$. A continuous solution of the Einstein equations is obtained by first rewriting (A.1) as function of $x$ and $z$ and then analytic continutate to the $x<0$ region. In this way, we get smooth $g(z, x)$ as (3.6). The solution is parametrized by two free parameters $\alpha_{1}$ and $\alpha_{2}$.

Next we solve (3.2) for the embedding function of $Q$ in the above background. We obtain, for $d=3$, the results

$$
x=-\sinh (\rho) z+\frac{k \cosh ^{2} \rho}{2(d-1)} z^{2}+c_{3} z^{3}+O\left(k^{3}\right)
$$

with $c_{3}$ given by

$$
\begin{aligned}
c_{3}=-\frac{\sinh \rho}{24}[ & 7 k_{1}^{2}+4 k_{2} k_{1}+7 k_{2}^{2}-4\left(q_{1}+q_{2}\right) \\
& +\left(5 k_{1}^{2}+2 k_{2} k_{1}+5 k_{2}^{2}-2\left(q_{1}+q_{2}\right)\right) \cosh (2 \rho) \\
& \left.+\alpha_{1}^{2}\left(k_{1}-k_{2}\right)^{2}\left((2+\cosh (2 \rho)) \log \left(\operatorname{coth}^{2} \rho\right)-1\right)\right] .
\end{aligned}
$$

The boundary conditions (3.2) also restrict solutions (3.6) and fix the integral constants to be

$$
\alpha_{1}=\frac{-1}{\theta}, \quad \alpha_{2}=-\frac{\alpha_{1}}{2} k^{2},
$$

where $\theta=\frac{\pi}{2}+2 \tan ^{-1}\left(\tanh \left(\frac{\rho}{2}\right)\right)$ is the angle between $M$ and the bulk boundary $Q$. It should be mentioned that, following our method, the above $\alpha_{1}$ is independently obtained in a recent paper [34]. The derivation of (A.4)-(A.6) is straightforward. For simplicity, let us first focus on the leading order $O(k)$ term. From dimensional analysis, the embedding function of $Q$ takes the form $x=-\sinh (\rho) z+c_{2} k z^{2}+O\left(k^{2}\right)$ with $c_{2}$ a dimensionless constant. Substituting the metric (A.1) and the embedding function of $Q$ into the conditions (3.2), we get two independent equations at order $O(k)$

$$
\begin{aligned}
\operatorname{sech}^{5}(\rho)\left(-8 c_{2}+\cosh (2 \rho)+1\right) k & =0, \\
\left(\alpha_{1} \cosh ^{2}(\rho)\left(4 \tan ^{-1}\left(\tanh \frac{\rho}{2}\right)+\pi\right)+8 c_{2}\right) \bar{k}_{a b} & =0 .
\end{aligned}
$$

Solving the above equations, we obtain $c_{2}$ and $\alpha_{1}$ as shown in (A.4), (A.6). Similarly, we obtain $c_{3}$ and $\alpha_{2}$ from (3.2) at order $O\left(k^{2}\right)$. It is remarkable that the conditions (3.2) fix the bulk metric and embedding function of $Q$ at the same time.

Substituting (3.6), (A.1), (A.3), (A.6) into (3.7), we obtain the holographic stress tensor

$$
T_{i j}=\operatorname{diag}\left\{\frac{\alpha_{1}\left(k_{1}-k_{2}\right)^{2}}{x}, \frac{\alpha_{1}\left(k_{1}-k_{2}\right)}{x^{2}}-\frac{3 \alpha_{1}\left(k_{1}-k_{2}\right)^{2}}{2 x}, \frac{\alpha_{1}\left(k_{2}-k_{1}\right)}{x^{2}}-\frac{3 \alpha_{1}\left(k_{1}-k_{2}\right)^{2}}{2 x}\right\} .
$$


It is remarkable that all the $q_{a}$ dependence got cancelled away and the stress tensor (A.7) takes exactly the expected form (1.3) with coefficients as listed in table 3 . Recall that $k_{i j}$ in (1.3) is actually a tensor defined at $x$ instead of the boundary $x=0$. It can be obtained from parallel transport of the extrinsic curvature at $x=0$, i.e., $\bar{k}_{i j}(x)=g_{i}^{i^{\prime}} g_{j}^{j^{\prime}} \bar{k}_{i^{\prime} j^{\prime}}(0)=$ $\bar{k}_{i j}(0)-2 x k_{(i}^{l} \bar{k}_{j) l}+O\left(x^{2}\right)[8]$.

Further generalization of the our above results is possible. Let us discuss briefly the case of non-constant metric $h_{i j}(y)$ and extrinsic curvature $k_{i j}(y)$. In this case, $T_{i j}$ will include non-diagonal parts generally. These non-diagonal parts obey (1.3b) trivially, since by definition (3.7) $T_{i j}$ automatically satisfy the traceless and divergenceless conditions (1.2), which fixs the non-diagonal parts of stress tensor as (1.3b) completely.

Another generalization is to have more general boundary conditions of holographic BCFT by adding intrinsic curvatures on $Q$ [18]. For example, we consider

$$
I=\int_{N} \sqrt{G}(R-2 \Lambda)+2 \int_{Q} \sqrt{\gamma}\left(K-T-\lambda R_{Q}\right)
$$

with the Neumann boundary condition

$$
K_{\alpha \beta}-\left(K-T-\lambda R_{Q}\right) \gamma_{\alpha \beta}-2 \lambda R_{Q \alpha \beta}=0 .
$$

Substituting the solutions (3.6) into (A.9), we can solve the embedding function of $Q$ as (A.4) but with different parameter $c_{3}$ and different integration constants

$$
\begin{aligned}
\alpha_{1} & =\frac{1}{2 \lambda \operatorname{sech} \rho /(1-2 \lambda \tanh \rho)-\theta}, \\
\alpha_{2} & =-\frac{\alpha_{1}}{2} k^{2} .
\end{aligned}
$$

Here $T=2 \tanh \rho+2 \lambda \operatorname{sech}^{2}(\rho)$. From (3.7), we can derive the holographic stress tensor which takes exactly the expected form (1.3). It is remarkable that although the central charge $b_{2}=-\alpha_{1}$ changes, the relations (2.11) remain invariant for holographic BCFT with general boundary conditions. The above discussions can be generalized to higher dimensions easily. The $4 \mathrm{~d}$ solutions can be used to confirm the universal relations (2.13).

\section{A.2 4d BCFT}

Now Let us consider the case $d=4$. For simplicity, we also set $k_{a b}=\operatorname{diag}\left(k_{1}, k_{2}, k_{3}\right), q_{a b}=$ $\operatorname{diag}\left(q_{1}, q_{2}, q_{3}\right)$, where $k_{a}, q_{a}$ are constants. Substituting (A.1) into the Einstein equations, and using (A.2) to fix the integral constants, we obtain

$$
\begin{aligned}
& f(s)=1+2 \alpha_{1}-\frac{\alpha_{1}\left(s^{2}+2\right)}{\sqrt{s^{2}+1}}, \\
& X(s)=\frac{1}{6} s^{2}\left(2\left(q_{1}+q_{2}+q_{3}\right)-3\left(k_{1} k_{2}+k_{1} k_{3}+k_{3} k_{2}\right)\right)-\frac{1}{3}\left(k_{1}^{2}+k_{2}^{2}+k_{3}^{2}-k_{1} k_{2}-k_{1} k_{3}-k_{2} k_{3}\right) g_{1}(s), \\
& Q_{11}(s)=\frac{k_{1}^{2} g_{2}(s)+k_{2}^{2} g_{3}(s)+k_{1} k_{2} g_{4}(s)}{18\left(s^{2}+1\right)^{3 / 2}}, \\
&+\frac{1}{3 \sqrt{s^{2}+1}}\left[q_{1}\left(2 s^{2}+\sqrt{s^{2}+1}+2\right)+q_{2}\left(-s^{2}+\sqrt{s^{2}+1}-1\right)+3 \alpha_{2}\left(\left(\sqrt{s^{2}+1}-2\right) s^{2}+2\left(\sqrt{s^{2}+1}-1\right)\right)\right],
\end{aligned}
$$




$$
\begin{aligned}
& Q_{22}(s)=\frac{k_{2}^{2} g_{2}(s)+k_{1}^{2} g_{3}(s)+k_{2} k_{1} g_{4}(s)}{18\left(s^{2}+1\right)^{3 / 2}} \\
& +\frac{1}{3 \sqrt{s^{2}+1}}\left[q_{2}\left(2 s^{2}+\sqrt{s^{2}+1}+2\right)+q_{1}\left(-s^{2}+\sqrt{s^{2}+1}-1\right)+3 \alpha_{3}\left(\left(\sqrt{s^{2}+1}-2\right) s^{2}+2\left(\sqrt{s^{2}+1}-1\right)\right)\right], \\
& Q_{33}(s)= \\
& \quad-\frac{\left(k_{1}^{2}+k_{2}^{2}\right) g_{5}(s)+k_{1} k_{2} g_{6}(s)}{18\left(s^{2}+1\right)^{3 / 2}} \\
& \quad-\frac{\left(q_{1}+q_{2}\right)\left(s^{2}-\sqrt{s^{2}+1}+1\right)+3\left(\alpha_{2}+\alpha_{3}\right)\left(\left(\sqrt{s^{2}+1}-2\right) s^{2}+2\left(\sqrt{s^{2}+1}-1\right)\right)}{3 \sqrt{s^{2}+1}},
\end{aligned}
$$

where $g_{i}(s)$ are defined by

$$
\begin{aligned}
g_{1}(s)= & \alpha_{1}\left(\alpha_{1}\left(8 \sqrt{s^{2}+1}+s^{2}\left(\log \left(s^{2}+1\right)-4\right)-8\right)-2 s^{2}+4 \sqrt{s^{2}+1}-4\right)+s^{2} \\
g_{2}(s)= & 12\left(s^{2}+1\right)\left(-s^{2}+\sqrt{s^{2}+1}-1\right)+36 \alpha_{1}\left(s^{2}+1\right)\left(-s^{2}+2 \sqrt{s^{2}+1}-2\right) \\
& -\alpha_{1}^{2}\left(-86\left(\sqrt{s^{2}+1}-1\right)+s^{2}\left(22 s^{2}-71 \sqrt{s^{2}+1}+108\right)+6\left(s^{2}+1\right)^{3 / 2} \log \left(s^{2}+1\right)\right) \\
g_{3}(s)= & -6\left(s^{2}+1\right)\left(-s^{2}+\sqrt{s^{2}+1}-1\right)+6 \alpha_{1}\left(s^{2}+1\right)\left(-s^{2}+2 \sqrt{s^{2}+1}-2\right) \\
& +\alpha_{1}^{2}\left(14\left(\sqrt{s^{2}+1}-1\right)+s^{2}\left(2 s^{2}+11 \sqrt{s^{2}+1}-12\right)-6\left(s^{2}+1\right)^{3 / 2} \log \left(s^{2}+1\right)\right) \\
g_{4}(s)= & -12\left(s^{2}+1\right)\left(-s^{2}+\sqrt{s^{2}+1}-1\right)-30 \alpha_{1}\left(s^{2}+1\right)\left(-s^{2}+2 \sqrt{s^{2}+1}-2\right) \\
& +\alpha_{1}^{2}\left(22 s^{4}-86\left(\sqrt{s^{2}+1}-1\right)+s^{2}\left(108-71 \sqrt{s^{2}+1}\right)+6\left(s^{2}+1\right)^{3 / 2} \log \left(s^{2}+1\right)\right) \\
g_{5}(s)= & -6\left(s^{2}+1\right)\left(-s^{2}+\sqrt{s^{2}+1}-1\right)-6 \alpha_{1}\left(s^{2}+1\right)\left(\left(2 \sqrt{s^{2}+1}-3\right) s^{2}+2\left(\sqrt{s^{2}+1}-1\right)\right) \\
& +\alpha_{1}^{2}\left(44\left(\sqrt{s^{2}+1}-1\right)+8 s^{2}\left(7 \sqrt{s^{2}+1}-9\right)-6\left(s^{2}+1\right)^{3 / 2} \log \left(s^{2}+1\right)+s^{4}\left(15 \sqrt{s^{2}+1}-28\right)\right) \\
g_{6}(s)= & 3\left(s^{2}+1\right)\left(\left(3 \sqrt{s^{2}+1}-8\right) s^{2}+8\left(\sqrt{s^{2}+1}-1\right)\right) \\
& +12 \alpha_{1}\left(s^{2}+1\right)\left(\left(\sqrt{s^{2}+1}-3\right) s^{2}+4\left(\sqrt{s^{2}+1}-1\right)\right) \\
& +\alpha_{1}^{2}\left(4 s^{2}\left(\sqrt{s^{2}+1}-6\right)+28\left(\sqrt{s^{2}+1}-1\right)+6\left(s^{2}+1\right)^{3 / 2} \log \left(s^{2}+1\right)+s^{4}\left(4-15 \sqrt{s^{2}+1}\right)\right) .
\end{aligned}
$$

Note that since the full expressions of $Q_{a b}$ are too complicated, we only list the results with $k_{3}=q_{3}=0$ for $Q_{a b}$ in (A.12). We want to stress that we focus on the general case with nonzero $k_{3}$ and $q_{3}$, we just do not list the full expressions for simplicity.

The above solutions work well for $x>0$. A continuous solution of the Einstein equations is obtained by first rewriting (A.1), (A.11), (A.12), (A.13) as functions of $x$ and $z$ and then analytic continutate to the $x<0$ region. In fact, we only need to replace all $\sqrt{1+s^{2}}=\sqrt{1+\frac{z^{2}}{x^{2}}}$ in (A.11), (A.12), (A.13) by $\sqrt{x^{2}+z^{2}} / x$. One can check that after the analytic continutation, the metric (A.1) are solutions to Einstein equations for $x \in(-\infty, \infty)$. What is more, now it becomes continuous at $x=0$ (see $x f(s)$ as an example). The above solution is parametrized by three free parameters $\alpha_{1}, \alpha_{2}$ and $\alpha_{3}$.

Next we solve (3.2) for the embedding function of $Q$ in the above background. We obtain, for $d=4$, the results

$$
x=-\sinh (\rho) z+\frac{k \cosh ^{2} \rho}{2(d-1)} z^{2}+c_{3} z^{3}+O\left(k^{3}\right)
$$

with $c_{3}$ given by

$$
c_{3}=\frac{-1}{288} e^{-2 \rho} \sinh (\rho)\left[t_{1}\left(k_{1}^{2}+k_{2}^{2}+k_{3}^{2}\right)+t_{2}\left(k_{1} k_{2}+k_{1} k_{3}+k_{2} k_{3}\right)+t_{3}\left(q_{1}+q_{2}+q_{3}\right)\right],
$$


with $t_{i}$ given by

$$
\begin{aligned}
t_{1}= & 8+48 \sinh (2 \rho)+20 \sinh (4 \rho)+5 \log \left(\operatorname{coth}^{2}(\rho)\right) \\
& +\cosh (4 \rho)\left(\log \left(\operatorname{coth}^{2}(\rho)\right)+20\right)+\cosh (2 \rho)\left(6 \log \left(\operatorname{coth}^{2}(\rho)\right)+44\right) \\
t_{2}= & 16+24 \sinh (2 \rho)+4 \sinh (4 \rho)-5 \log \left(\operatorname{coth}^{2}(\rho)\right) \\
& -\cosh (4 \rho)\left(\log \left(\operatorname{coth}^{2}(\rho)\right)-4\right)+\cosh (2 \rho)\left(28-6 \log \left(\operatorname{coth}^{2}(\rho)\right)\right) \\
t_{3}= & -16 e^{2 \rho}(\cosh (2 \rho)+2) .
\end{aligned}
$$

The boundary conditions (3.2) also fix all the integral constants of the solutions (A.1), (A.11), (A.12), (A.13)

$$
\begin{aligned}
& \alpha_{1}=\frac{-1}{2(\tanh (\rho)+1)}, \\
& \begin{aligned}
\alpha_{2}=\frac{-1}{144(\sinh (\rho)+\cosh (\rho))^{2}} & {\left[35 k_{1}^{2}+25 k_{2} k_{1}+25 k_{3} k_{1}-37 k_{2}^{2}-37 k_{3}^{2}-11 k_{2} k_{3}-24 q_{1}+12 q_{2}+12 q_{3}\right.} \\
& +4\left(4 k_{1}^{2}-7\left(k_{2}+k_{3}\right) k_{1}-5 k_{2}^{2}-5 k_{3}^{2}+2 k_{2} k_{3}-6 q_{1}+3 q_{2}+3 q_{3}\right) \sinh (2 \rho) \\
& \left.+3\left(k_{1}^{2}-5\left(k_{2}+k_{3}\right) k_{1}-7 k_{2}^{2}-7 k_{3}^{2}-k_{2} k_{3}-8 q_{1}+4 q_{2}+4 q_{3}\right) \cosh (2 \rho)\right]
\end{aligned} \\
& \begin{aligned}
\alpha_{3}=\alpha_{2}\left[k_{1} \leftrightarrow k_{2}, q_{1} \leftrightarrow q_{2}\right] . & \text { (A.17) }
\end{aligned}
\end{aligned}
$$

It should be mentioned that, following our method, the above $\alpha_{1}$ is independently obtained in a recent paper [34], which exactly agrees with our results when using our notations. The derivation of (A.14)-(A.17) is straightforward. For simplicity, let us first focus on the leading order $O(k)$ term. From dimensional analysis, the embedding function of $Q$ takes the form $x=-\sinh (\rho) z+c_{2} k z^{2}+O\left(k^{2}\right)$ with $c_{2}$ a dimensionless constant. Substituting the metric (A.1) and the embedding function of $Q$ into the conditions (3.2), we get two independent equations at order $O(k)$

$$
\begin{aligned}
\operatorname{sech}^{5}(\rho)\left(-12 c_{2}+\cosh (2 \rho)+1\right) k & =0, \\
4 k_{1}\left(\alpha_{1}(\sinh (2 \rho)+\cosh (2 \rho)+1)+6 c_{2}\right) & \\
-\left(k_{2}+k_{3}\right)\left(2 \alpha_{1}(\sinh (2 \rho)+\cosh (2 \rho)+1)+3\left(-8 c_{2}+\cosh (2 \rho)+1\right)\right) & =0 .
\end{aligned}
$$

Solving the above equations, we obtain $c_{2}$ and $\alpha_{1}$ as shown in (A.14), (A.17)

$$
c_{2}=\frac{\cosh ^{2}(\rho)}{6}, \quad \alpha_{1}=\frac{-1}{2(\tanh (\rho)+1)} .
$$

Similarly, we can obtain $c_{3}, \alpha_{2}, \alpha_{3}$ from Neumann boundary conditions (3.2) at the next order $O\left(k^{2}, q\right)$. It is remarkable that the conditions (3.2) fix the bulk metric and embedding function of $Q$ at the same time.

Substituting the solutions (A.1), (A.11), (A.12), (A.13), (A.17) into the formula

$$
T_{i j}=\lim _{z \rightarrow 0} d \frac{\delta g_{i j}}{z^{d}},
$$

and noting BCFT is defined in $x \in[0, \infty)$, we obtain the holographic stress tensor with non-zero components given by 


$$
\begin{aligned}
T_{x x}= & -\frac{k_{1}^{2}+k_{2}^{2}+k_{3}^{2}-k_{1} k_{2}-k_{1} k_{3}+k_{2} k_{3}}{3 x^{2}(\tanh (\rho)+1)} \\
T_{11}= & -\frac{\left(2 k_{1}-k_{2}-k_{3}\right)}{3(1+\tanh \rho) x^{3}} \\
& +\frac{\cosh (\rho)-\sinh (\rho)}{18 x^{2}}\left[2 k_{1}^{2}(5 \sinh (\rho)+8 \cosh (\rho))+\left(k_{2}^{2}+k_{3}^{2}\right)(7 \cosh (\rho)-5 \sinh (\rho))\right. \\
& \left.+2 k_{2} k_{3}(\sinh (\rho)+4 \cosh (\rho))-k_{1}\left(k_{2}+k_{3}\right)(\sinh (\rho)+19 \cosh (\rho))+3\left(q_{2}+q_{3}\right) \sinh (\rho)-6 q_{1} \sinh (\rho)\right], \\
T_{22}= & T_{11}\left[k_{1} \leftrightarrow k_{2}, q_{1} \leftrightarrow q_{2}\right], \\
T_{33}= & T_{11}\left[k_{1} \leftrightarrow k_{3}, q_{1} \leftrightarrow q_{3}\right] .
\end{aligned}
$$

We can rewrite the above holographic stress tensor into convariant form:

$T_{i j}=\frac{2 \alpha_{1}\left(\bar{k}_{i j}-2 x k_{(i}^{l} \bar{k}_{j) l}\right)}{x^{3}}+\frac{\alpha_{1}\left(n_{i} n_{j}-\frac{h_{i j}}{3}\right) \operatorname{Tr} \bar{k}^{2}}{x^{2}}+\frac{p_{1} C_{i k j l} n^{k} n^{l}+p_{2} k \bar{k}_{i j}+p_{3}\left(k_{i l} k_{j}^{l}-\frac{1}{3} h_{i j} \operatorname{Tr} k^{2}\right)}{x^{2}}$

where $^{-}$means traceless parts, $C_{i k j l} n^{k} n^{l}=-\frac{1}{2} \bar{q}_{i j}+\frac{1}{2} k \bar{k}_{i j}, \alpha_{1}$ is given by (A.17), $n_{i}=$ $(-1,0,0,0), h_{i j}=\operatorname{diag}(0,1,1,1)$ and $p_{i}$ are given by

$$
p_{1}=p_{3}=\frac{\tanh (\rho)}{\tanh (\rho)+1}, \quad p_{2}=\frac{-4 \tanh (\rho)-5}{6(\tanh (\rho)+1)} .
$$

Now let us turn to the field theoretical result of BCFT stress tensor (1.3a), (1.3b), (1.4), which takes the form

$$
T_{i j}=\frac{2 \alpha_{1}\left(\bar{k}_{i j}-2 x k_{(i}^{l} \bar{k}_{j) l}\right)}{x^{3}}+\frac{\alpha_{1}\left(n_{i} n_{j}-\frac{h_{i j}}{3}\right) \operatorname{Tr} \bar{k}^{2}}{x^{2}}+\frac{\beta_{1} C_{i k j l} n^{k} n^{l}+\beta_{3} k \bar{k}_{i j}+\beta_{4}\left(k_{i l} k_{j}^{l}-\frac{1}{3} h_{i j} \operatorname{Tr} k^{2}\right)}{x^{2}}
$$

Recall that $k_{i j}$ in eqs. (1.3a), (1.3b), (1.4) is actually a tensor defined at $x$ instead of the boundary $x=0$. It can be obtained from parallel transport of the extrinsic curvature at $x=0$, i.e., $\bar{k}_{i j}(x)=g_{i}^{i^{\prime}} g_{j}^{j^{\prime}} \bar{k}_{i^{\prime} j^{\prime}}(0)=\bar{k}_{i j}(0)-2 x k_{(i}^{l} \bar{k}_{j) l}+O\left(x^{2}\right)[8]$.

Comparing the holographic stress tensor (A.22) with the field theoretical result (A.24), we get

$$
\beta_{1}=p_{1}=\frac{\tanh (\rho)}{\tanh (\rho)+1}, \beta_{3}=p_{2}=\frac{-4 \tanh (\rho)-5}{6(\tanh (\rho)+1)}, \beta_{4}=p_{3}=\frac{-4 \tanh (\rho)-5}{6(\tanh (\rho)+1)} .
$$

Now it is easy to check that the Casimir coefficients $\alpha_{1}, \beta_{i}$ indeed satisfy the universal relations

$$
\begin{aligned}
& \alpha_{1}=\frac{b_{4}}{2}, \quad \beta_{1}=\frac{c}{2 \pi^{2}}+b_{4}, \quad \beta_{2}=0, \\
& \beta_{3}=2 b_{3}+\frac{13}{6} b_{4}, \quad \beta_{4}=-3 b_{3}-2 b_{4} .
\end{aligned}
$$

provided the boundary central charges are given by

$$
b_{3}=\frac{1}{1+\tanh \rho}-\frac{1}{3}, \quad b_{4}=\frac{-1}{1+\tanh \rho},
$$

Since we have four relations and two unknown variables, this is a non-trivial check of the universal relations (A.26). Recall that in (A.26) we have $c=2 \pi^{2}$ for Einstein gravity 
$\left(16 \pi G_{N}=1\right)$. In fact, the central charges (A.27) can be independently derived from the logarithmic divergent term of action by using the perturbation solution of order $O\left(k^{3}\right)$. It should be mentioned that the holographic results do not test the relations $\beta_{2}=0$, since in our setup we have $h_{a b}=\delta_{a b}$ and thus $\mathcal{R}_{i j}=0$ in the stress tensor eq. (4) of the revised letter. However, this is a trivial relation and there is no need to test it. From the conformal symmetry, [8] finds that there is no $\bar{R}_{i j}=\overline{\mathcal{R}}_{i j}+O\left(k^{2}, q\right)$ terms in the stress tensor. As a result, we must have $\beta_{2}=0$.

Further generalization of the our above results is possible. Let us discuss briefly the case of non-constant metric $h_{i j}(y)$ and extrinsic curvature $k_{i j}(y)$. In this case, $T_{i j}$ will include non-diagonal parts generally. These non-diagonal parts obey eq. (3b) of the revised letter trivially, since by definition (A.20) $T_{i j}$ automatically satisfy the traceless and divergenceless conditions, which fixs the non-diagonal parts of stress tensor as eq. (3b) in the revised letter completely.

Now we have shown that the holographic BCFT indeed obeys the universal relations (2.13), (A.26) between Casimir coefficients and central charges.

Open Access. This article is distributed under the terms of the Creative Commons Attribution License (CC-BY 4.0), which permits any use, distribution and reproduction in any medium, provided the original author(s) and source are credited.

\section{References}

[1] H.B.G. Casimir, On the attraction between two perfectly conducting plates, Indag. Math. 10 (1948) 261 [Kon. Ned. Akad. Wetensch. Proc. 51 (1948) 793] [Front. Phys. 65 (1987) 342] [Kon. Ned. Akad. Wetensch. Proc. 100N3-4 (1997) 61] [INSPIRE].

[2] G. Plunien, B. Müller and W. Greiner, The Casimir effect, Phys. Rept. 134 (1986) 87 [INSPIRE].

[3] M. Bordag, U. Mohideen and V.M. Mostepanenko, New developments in the Casimir effect, Phys. Rept. 353 (2001) 1 [quant-ph/0106045] [INSPIRE].

[4] T. Appelquist and A. Chodos, The quantum dynamics of Kaluza-Klein theories, Phys. Rev. D 28 (1983) 772 [INSPIRE].

[5] T. Appelquist and A. Chodos, Quantum effects in Kaluza-Klein theories, Phys. Rev. Lett. 50 (1983) 141 [INSPIRE].

[6] K.A. Milton, The Casimir effect: recent controversies and progress, J. Phys. A 37 (2004) R209 [hep-th/0406024] [INSPIRE].

[7] V.V. Dodonov, Current status of the dynamical Casimir effect, Phys. Scripta 82 (2010) 038105 [INSPIRE].

[8] D. Deutsch and P. Candelas, Boundary effects in quantum field theory, Phys. Rev. D 20 (1979) 3063 [INSPIRE].

[9] A. Lewkowycz and E. Perlmutter, Universality in the geometric dependence of Rényi entropy, JHEP 01 (2015) 080 [arXiv:1407.8171] [INSPIRE].

[10] X. Dong, Shape dependence of holographic Rényi entropy in conformal field theories, Phys. Rev. Lett. 116 (2016) 251602 [arXiv: 1602.08493] [INSPIRE]. 
[11] K. Jensen and A. O'Bannon, Constraint on defect and boundary renormalization group flows, Phys. Rev. Lett. 116 (2016) 091601 [arXiv: 1509.02160] [INSPIRE].

[12] D. Fursaev, Conformal anomalies of CFT's with boundaries, JHEP 12 (2015) 112 [arXiv: 1510.01427] [INSPIRE].

[13] C.P. Herzog, K.-W. Huang and K. Jensen, Universal entanglement and boundary geometry in conformal field theory, JHEP 01 (2016) 162 [arXiv:1510.00021] [INSPIRE].

[14] G. Kennedy, R. Critchley and J.S. Dowker, Finite temperature field theory with boundaries: stress tensor and surface action renormalization, Annals Phys. 125 (1980) 346 [INSPIRE].

[15] G. Kennedy, Finite temperature field theory with boundaries: the photon field, Annals Phys. 138 (1982) 353 [INSPIRE].

[16] T. Takayanagi, Holographic dual of BCFT, Phys. Rev. Lett. 107 (2011) 101602 [arXiv: 1105.5165] [INSPIRE].

[17] R.-X. Miao, C.-S. Chu and W.-Z. Guo, New proposal for a holographic boundary conformal field theory, Phys. Rev. D 96 (2017) 046005 [arXiv: 1701.04275] [INSPIRE].

[18] C.-S. Chu, R.-X. Miao and W.-Z. Guo, On new proposal for holographic BCFT, JHEP 04 (2017) 089 [arXiv: 1701.07202] [INSPIRE].

[19] W. Song, Q. Wen and J. Xu, Generalized gravitational entropy for warped anti-de Sitter space, Phys. Rev. Lett. 117 (2016) 011602 [arXiv:1601.02634] [INSPIRE].

[20] M. Nozaki, T. Takayanagi and T. Ugajin, Central charges for BCFTs and holography, JHEP 06 (2012) 066 [arXiv: 1205.1573] [INSPIRE].

[21] S. de Haro, S.N. Solodukhin and K. Skenderis, Holographic reconstruction of space-time and renormalization in the AdS/CFT correspondence, Commun. Math. Phys. 217 (2001) 595 [hep-th/0002230] [INSPIRE].

[22] J.S. Dowker and G. Kennedy, Finite temperature and boundary effects in static space-times, J. Phys. A 11 (1978) 895 [inSPIRE].

[23] T. Emig, A. Hanke, R. Golestanian and M. Kardar, Probing the strong boundary shape dependence of the Casimir force, Phys. Rev. Lett. 87 (2001) 260402 [cond-mat/0106028] [INSPIRE].

[24] M. Schaden, Dependence of the direction of the Casimir force on the shape of the boundary, Phys. Rev. Lett. 102 (2009) 060402 [InSPIRE].

[25] M.A. Rajabpour, Classification of the sign of the critical Casimir force in two dimensional systems at asymptotically large separations, Phys. Rev. D 94 (2016) 105029 [arXiv: 1609.06279] [INSPIRE].

[26] J.L. Cardy, Boundary conformal field theory, hep-th/0411189 [INSPIRE].

[27] D.M. McAvity and H. Osborn, Energy momentum tensor in conformal field theories near a boundary, Nucl. Phys. B 406 (1993) 655 [hep-th/9302068] [INSPIRE].

[28] J. Cardy, private communication.

[29] M. Billò, V. Gonçalves, E. Lauria and M. Meineri, Defects in conformal field theory, JHEP 04 (2016) 091 [arXiv: 1601.02883] [INSPIRE].

[30] C.-S. Chu and R.-X. Miao, Universality in the shape dependence of holographic Rényi entropy for general higher derivative gravity, JHEP 12 (2016) 036 [arXiv:1608.00328] [INSPIRE]. 
[31] L. Bianchi, S. Chapman, X. Dong, D.A. Galante, M. Meineri and R.C. Myers, Shape dependence of holographic Rényi entropy in general dimensions, JHEP 11 (2016) 180 [arXiv: 1607.07418] [INSPIRE].

[32] L. Bianchi, M. Meineri, R.C. Myers and M. Smolkin, Rényi entropy and conformal defects, JHEP 07 (2016) 076 [arXiv: 1511.06713] [INSPIRE].

[33] S. Balakrishnan, S. Dutta and T. Faulkner, Gravitational dual of the Rényi twist displacement operator, Phys. Rev. D 96 (2017) 046019 [arXiv:1607.06155] [INSPIRE].

[34] D. Seminara, J. Sisti and E. Tonni, Corner contributions to holographic entanglement entropy in $A d S_{4} / B C F T_{3}, J H E P 11$ (2017) 076 [arXiv: 1708.05080] [INSPIRE]. 